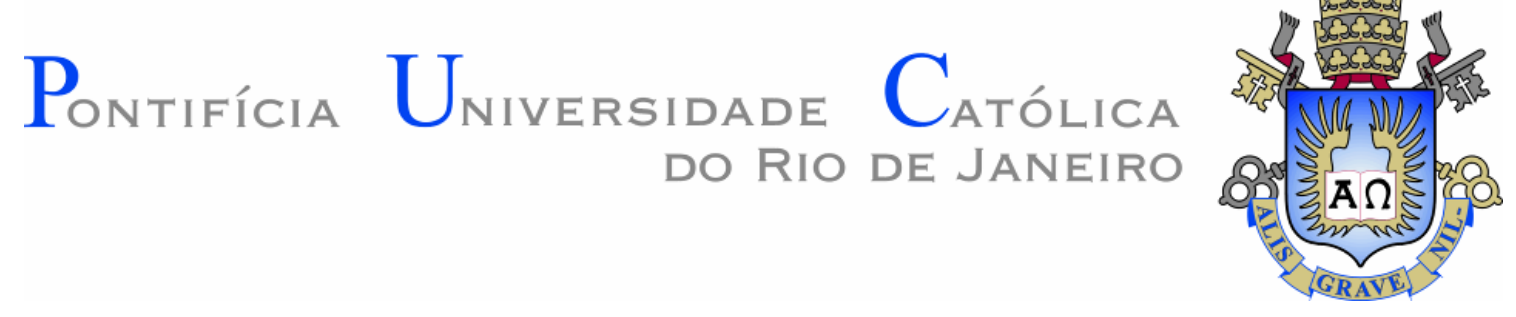

Roberta de Barros do Rego Macedo

\title{
O Instituto de Pesquisas Educacionais do Distrito Federal \\ nos anos de 1930
}

Tese apresentada como requisito parcial para obtenção do grau de Doutor pelo Programa de Pós-graduação em Educação do Departamento de Educação da PUC-Rio

Orientador: Profa. Ana Waleska Pollo C. Mendonça 


\section{Pontifícia Universidade Católica $_{\text {a }}$

Roberta de Barros do Rego Macedo

\section{O Instituto de Pesquisas Educacionais do Distrito Federal nos anos de 1930}

Tese apresentada como requisito parcial para obtenção do grau de Doutor pelo Programa de Pós-graduação em Educação do Departamento de Educação do Centro de Teologia e Ciências Humanas da PUC-Rio. Aprovada pela Comissão Examinadora abaixo assinada.

Prof $^{a}$ Ana Waleska Pollo Campos Mendonça

Orientadora

Departamento de Educação - PUC-Rio

Prof $^{\mathrm{a}}$ Patrícia Coelho da Costa

Presidente

Departamento de Educação - PUC-Rio

Prof $^{\circ}$ André Luiz Paulilo

UNICAMP

Prof $^{\mathrm{a}}$ Sonia Maria de Castro Nogueira Lopes

UFRJ

Prof $^{\mathrm{a}}$ Miriam Waidenfeld Chaves

UFRJ

Prof $^{a}$ Denise Berruezzo Portinari

Coordenador Setorial do Centro de teologia e Ciências

Humanas

PUC-Rio

Rio de Janeiro, 15 de Abril de 2013 
Todos os direitos reservados. É proibida a reprodução total ou parcial do trabalho sem autorização da universidade, da autora e da orientadora.

\section{Roberta de Barros do Rego Macedo}

Graduou-se em Pedagogia pela Universidade do Estado do Rio de Janeiro (2000) e obteve o título de mestre em Educação pela PUC-Rio, em 2007. Atualmente trabalha como Orientadora Educacional na Secretaria Municipal de Educação de Duque de Caxias e é coordenadora pedagógica do Colégio Israelita A.Liessin.

Ficha Catalográfica

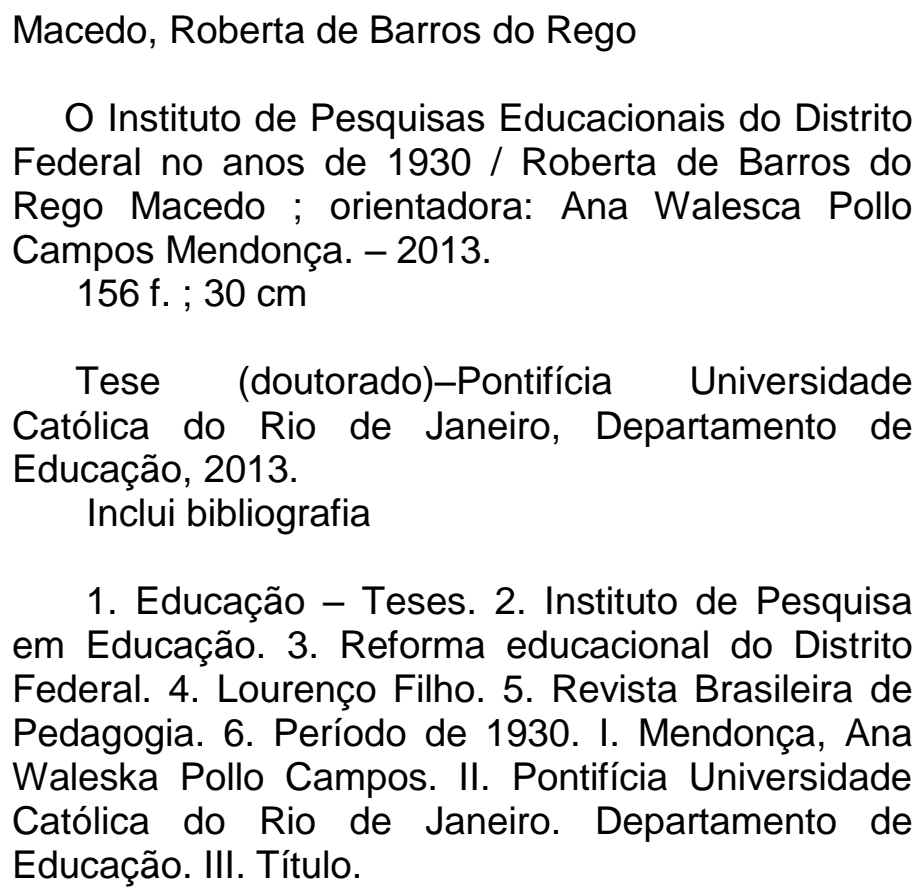

1. Educação - Teses. 2. Instituto de Pesquisa em Educação. 3. Reforma educacional do Distrito Federal. 4. Lourenço Filho. 5. Revista Brasileira de Pedagogia. 6. Período de 1930. I. Mendonça, Ana Waleska Pollo Campos. II. Pontifícia Universidade Católica do Rio de Janeiro. Departamento de Educação. III. Título. 
“Ainda que eu falasse a língua dos homens e falasse a língua dos anjos, sem amor eu nada seria". Aos amores da minha vida, Yuri e Bruno. 


\section{Agradecimentos}

A minha orientadora professora Ana Waleska pela paciência e estimulo para a realização desse trabalho.

A PUC-Rio, pelos auxílios concedidos, sem os quais esse trabalho não poderia ter sido realizado.

Aos meus pais pelos erros e acertos que possibilitaram a minha formação.

A Annamaria Wolter pela admiração, estimulo e credibilidade que sempre depositou.

A Daniella e Sue pela a atenção e carinho de todas as horas.

As minhas amigas Bernadete e Thalita pela parceria, carinho e acolhimento.

A todos os funcionários da PUC-Rio pela ajuda.

A todos os professores do Departamento de Educação PUC-Rio pelos ensinamentos. 


\section{Resumo}

Macedo, Roberta de Barros do Rego; Mendonça, Ana Waleska Pollo Campos. O Instituto de Pesquisas Educacionais do Distrito Federal nos anos de 1930. Rio de Janeiro, 2013. 156p Tese de Doutorado Departamento de Educação, Pontifícia Universidade Católica do Rio de Janeiro.

Trata-se de uma pesquisa que tem como objetivo estudar o Instituto de Pesquisas Educacionais (IPE), do Departamento de Educação do Rio de Janeiro, na década de 1930. Com o apoio do prefeito Pedro Ernesto, Anísio Teixeira desenvolveu uma política de reformulação da educação, no Rio de Janeiro, que teve como uma das referências um conjunto de iniciativas o IPE. No período, o projeto de muitos intelectuais que fomentavam os debates educacionais era viabilizar, por intermédio da educação, a reconstrução nacional do Brasil. A educação escolar era destinada a uma parcela pequena da sociedade e, com isso, transparecia seu caráter elitista, que dificultava o processo de democratização almejada por muitos brasileiros na época. O IPE inicia as suas atividades no ano de 1933 com o objetivo de tratar da investigação e da formulação dos planos de trabalho dos professores, assim como dos programas, métodos e processos educacionais. Sua trajetória assume um rumo diferente em 1937, quando Getúlio Vargas, em novembro, outorga uma nova constituição e instala o Estado Novo. Alguns indicadores apontam para particularidades que tornam o IPE um objeto interessante de estudo, principalmente ao considerarmos o contexto político dos anos em que se manteve em funcionamento fiel ao seu projeto inicial; a sucessão de gestores após o ano de 1935, ao término da gestão de Anísio Teixeira, e as relações entre sua experiência e o surgimento do Instituto Nacional de Estudos e Pesquisas Educacionais (Inep), no ano de 1937. Por intermédio dos documentos que compõem a história deste Instituto, chama-se a atenção para uma visão mais detalhada acerca da trajetória da instituição que funcionou como eixo norteador da Reforma da Instrução Pública do antigo Distrito Federal e, posteriormente, como um lócus de amálgamas de distintos projetos educacionais até 1940, ano em que muda a sua denominação e seu estatuto de funcionamento. 


\section{Palavras-chave}

Instituto de Pesquisa em Educação; Reforma Educacional do Distrito Federal; Lourenço Filho; Revista Brasileira de Pedagogia; Década de 1930. 


\section{Abstract}

Macedo, Roberta de Barros do Rego; Mendonça, Ana Waleska Pollo Campos (Advisor). The Institute for Educational Research in the Federal District during the 1930s. Rio de Janeiro, 2013. 156p. Doctoral Thesis - Departamento de Educação, Pontifícia Universidade Católica do Rio de Janeiro.

The objective of this paper is to study the Institute for Educational Research (IPE) of the Education Department in Rio de Janeiro in the 1930s. Having the support of the mayor, Pedro Ernesto, Anisio Teixeira developed a politics for the reformulation in education, in Rio de Janeiro, based on a reference point and initiatives presented by the IPE. During this period, it was viable for many intellectuals to debate the education as means for national reconstruction. Schooling was destined for a small number of people in society, characterized by the elite members, which made it difficult for the democratic process desired by many Brazilians at the time. IPE began its activities in 1933 with the objective of investigating and building of teachers work such as programs, methods and educational process. A new direction was taken in November 1937 when Getulio Vargas officialized a new constitution installed in the new state. Some specific indicators turn IPE into an interesting object of study, especially considering the political context that maintained a loyalty to the initial project; the success of administrators after 1935, at the end of Anisio Teixeira's term, and the relations between his experience and rise of the Institute of National Research in Education (Inep) in 1937. Through documents that compose the history of the above mentioned institute, one may note a more detailed vision of its history as a guideline for the Reform of Public Instruction of the old Federal District, and later as the focus for specific educational projects up to 1940, the year in which the changes in denomination and working policies happened.

\section{Keywords}

Institute for Educational Research; Educational Reform of Federal District; Lourenço Filho; Brazilian Journal of Pedagogy; 1930's decade 


\section{Résumé}

Macedo, Roberta de Barros do Rego; Mendonça, Ana Waleska Pollo Campos (Leader). L'INSTITUT DE RECHERCHE en ÉDUCATION dans le District fédéral durant les années 1930. Rio de Janeiro, 2013, 156p. Thése de Doctorat - Departamento de Educação, Pontifícia Universidade Católica do Rio de Janeiro.

L'objectif de cet article est d'étudier l'Institut de Recherche en Éducation (IPE) du ministère de l'Éducation à Rio de Janeiro dans les années 1930. Avoir le soutien du maire, Pedro Ernesto, Anisio Teixeira a développé une politique pour la reformulation de l'enseignement, à Rio de Janeiro, basée sur un point et initiatives présentés par l'IPE référence. Pendant cette période, il était viable pour de nombreuses intelectuals pour débattre de l'éducation comme moyen de reconstruction nationale. L'école était destiné à un petit nombre de personnes dans la société, caractérisé par les membres de l'élite, ce qui fait qu'il est difficile pour le processus démocratique souhaité par de nombreux Brésiliens à l'époque. IPE a commencé ses activités en 1933 avec l'objectif d'enquêter et de construction des enseignants fonctionnent comme des programmes, des méthodes et processus éducatif. Une nouvelle direction a été prise en Novembre 1937 où Getulio Vargas a officialisé une nouvelle constitution installé dans le Nouvel Etat. Certains indicateurs spécifiques tournent IPE en un objet d'étude intéressant, surtout compte tenu du contexte politique qui a maintenu une loyauté envers le projet initial, le succès des administrateurs après 1935, à la fin du mandat de Anisio Teixeira, et les relations entre son expérience et son lieu de l'Institut de recherche national en éducation (Inep) en 1937. Grâce à des documents qui composent l'histoire de l'institut susmentionné, on peut noter une vision plus détaillée de son histoire comme un guide pour la réforme de l'Instruction publique de l'ancien District fédéral, et plus tard comme la mise au point des projets éducatifs spécifiques jusqu'en 1940, l'année où les changements de dénomination et les politiques de travail ont eu lieu. 


\section{Mots Clefs}

Institut de Recherche en Éducation; réforme de l'éducation du District federal; Lourenço Filho; Journal brésilien de l'éducation; 1930 Période 


\section{Sumário}

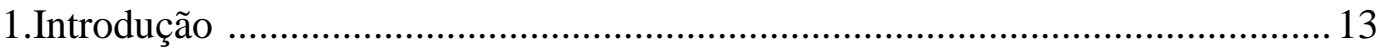

2. A configuração educacional do Rio de Janeiro nos anos de 1930 ...................20

2.1. A reorganização do campo educacional nas décadas de 1920 e 1930 ............. 29

2.2. Antecedentes da Reforma de Anísio Teixeira no DF .................................... 34

2.3. A Reforma da Instrução Pública de 1932 ........................................................ 37

3. O Instituto de Pesquisas Educacionais: do projeto à prática ..........................45

3.1. "Bem pouco falta sem nada sobrar": a reorganização do Departamento de

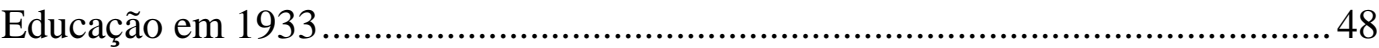

3.2. Um “órgão vital” para o sistema de ensino do DF ..........................................49

4. Do IPE ao Inep: o fio condutor de Lourenço Filho ..........................................61

4.1. A questão da incorporação do Instituto à UDF: o poder ameaçado ................66 65

4.2. "O terrível lema que vale realizar, mesmo que imperfeitamente": Lourenço

Filho e o projeto educacional do ministro Gustavo Capanema............................69 69

4.3. O surgimento do Inep e o apagar de uma memória....................................... 82

5. A Gestão de Everardo Backeuser: a presença do ideário católico no IPE ..... 94

5.1. O IPE na Revista Brasileira de Pedagogia .................................................. 96

5.2. A Escola México: uma escola laboratório para os católicos ......................... 108

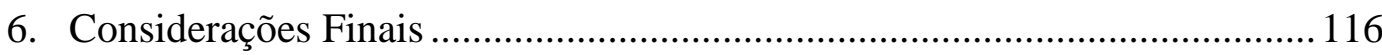

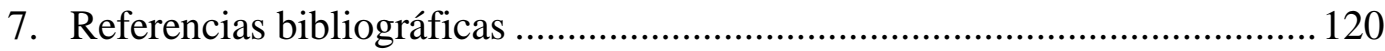

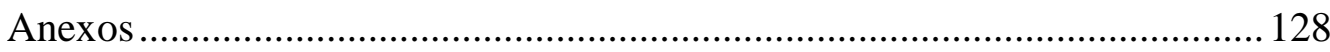




\section{Abreviaturas}

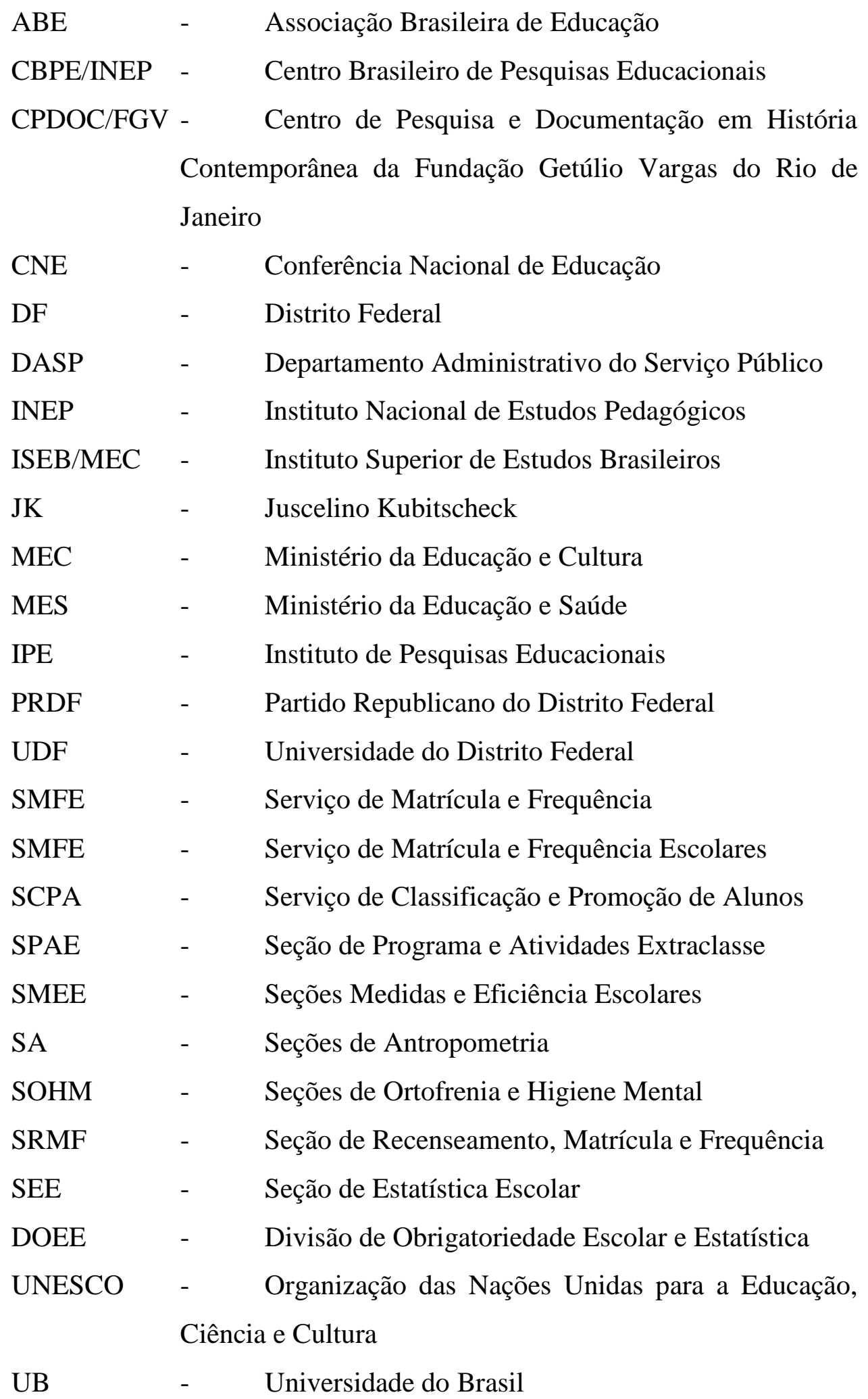

